\title{
M3MS-16OR0401086 - REPORT ON NEAMS WORKBENCH SUPPORT FOR MOOSE APPLICATIONS
}
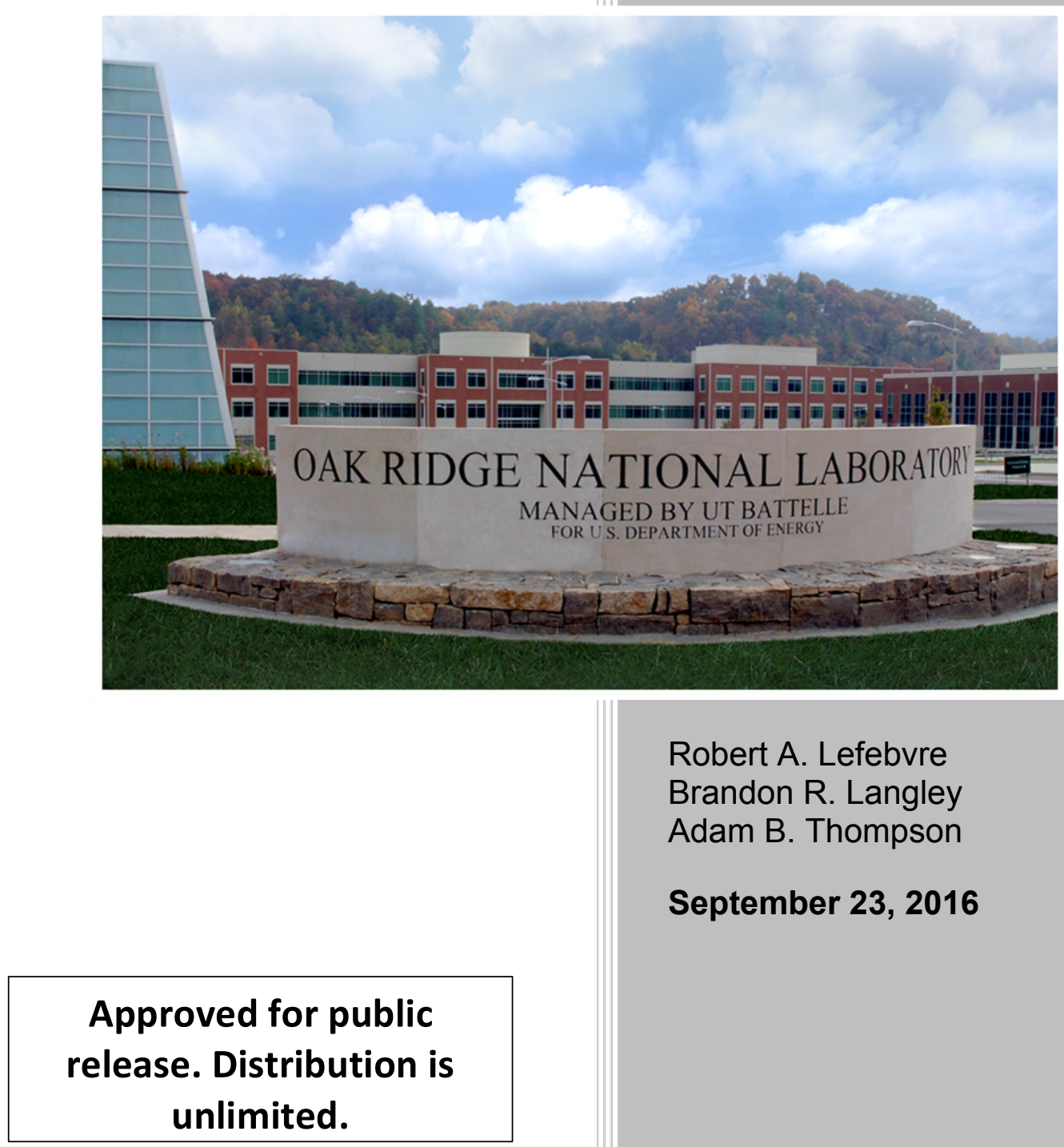

Robert A. Lefebvre Brandon R. Langley Adam B. Thompson

September 23, 2016 


\section{DOCUMENT AVAILABILITY}

Reports produced after January 1, 1996, are generally available free via US Department of Energy (DOE) SciTech Connect.

Website http://www.osti.gov/scitech/

Reports produced before January 1, 1996, may be purchased by members of the public from the following source:

National Technical Information Service
5285 Port Royal Road
Springfield, VA 22161
Telephone 703-605-6000 (1-800-553-6847)
TDD 703-487-4639
Fax 703-605-6900
E-mail info@ntis.gov
Website http://www.ntis.gov/help/ordermethods.aspx

Reports are available to DOE employees, DOE contractors, Energy Technology Data Exchange representatives, and International Nuclear Information System representatives from the following source:

Office of Scientific and Technical Information

PO Box 62

Oak Ridge, TN 37831

Telephone 865-576-8401

Fax 865-576-5728

E-mail reports@osti.gov

Website http://www.osti.gov/contact.html

This report was prepared as an account of work sponsored by an agency of the United States Government. Neither the United States Government nor any agency thereof, nor any of their employees, makes any warranty, express or implied, or assumes any legal liability or responsibility for the accuracy, completeness, or usefulness of any information, apparatus, product, or process disclosed, or represents that its use would not infringe privately owned rights. Reference herein to any specific commercial product, process, or service by trade name, trademark, manufacturer, or otherwise, does not necessarily constitute or imply its endorsement, recommendation, or favoring by the United States Government or any agency thereof. The views and opinions of authors expressed herein do not necessarily state or reflect those of the United States Government or any agency thereof. 
Reactor and Nuclear Systems Division

\section{M3MS-16OR0401086 - Report on NEAMS Workbench Support for MOOSE Applications}

Robert A. Lefebvre

Brandon R. Langley

Adam B. Thompson

Date Published: September 2016

Prepared by

OAK RIDGE NATIONAL LABORATORY

Oak Ridge, TN 37831-6283

managed by

UT-BATTELLE, LLC

for the

US DEPARTMENT OF ENERGY

under contract DE-AC05-00OR22725 



\section{CONTENTS}

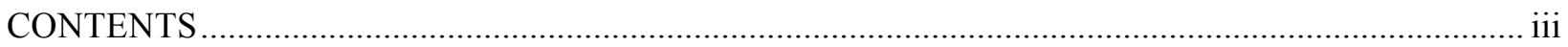

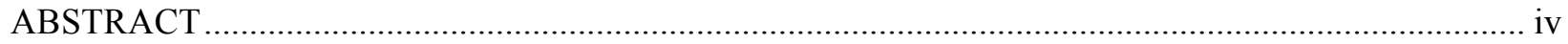

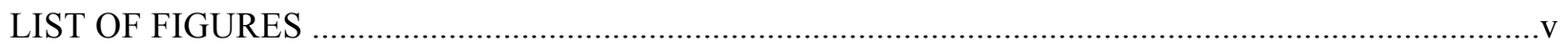

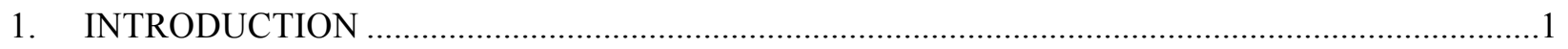

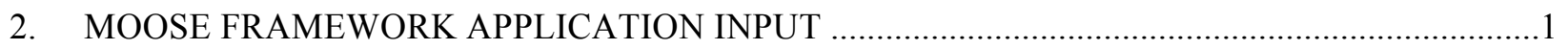

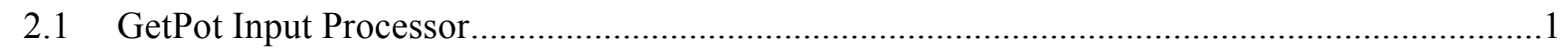

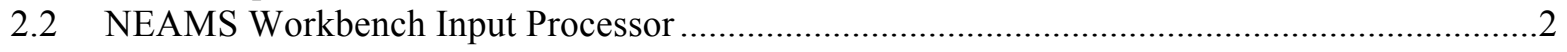

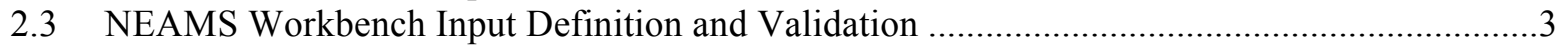

2.4 NEAMS Workbench MOOSE Input Creation ....................................................................

2.5 NEAMS Workbench MOOSE Input Considerations...........................................................

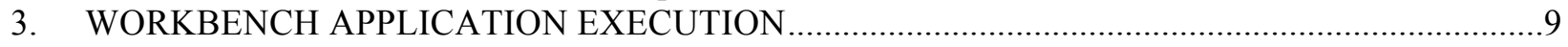

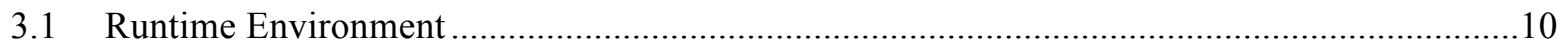

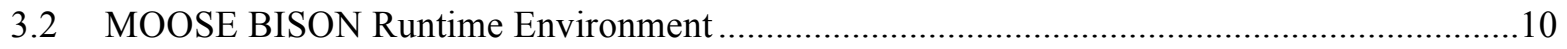

3.3 Future Runtime Environment Considerations.....................................................................11

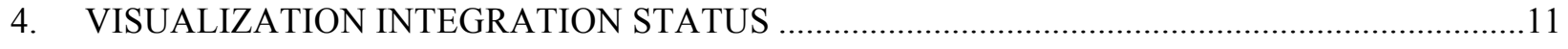

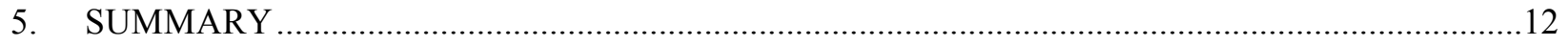




\begin{abstract}
This report summarizes the status of the Nuclear Energy Advanced Modeling and Simulation (NEAMS) Workbench from Oak Ridge National Laboratory (ORNL) and the integration of the MOOSE framework. This report marks the completion of NEAMS milestone M3MS-16OR0401086. This report documents the developed infrastructure to support the MOOSE framework applications, the applications' results, visualization status, the collaboration that facilitated this progress, and future considerations.
\end{abstract}




\section{LIST OF FIGURES}

Figure 1. GetPot Block, Sub Block, and Named Parameter Syntax. ....................................................

Figure 2, MOOSE Application Input Syntax Error Message Example. .................................................2

Figure 3. The NEAMS Workbench MOOSE BISON Input Quick Navigation Illustration........................

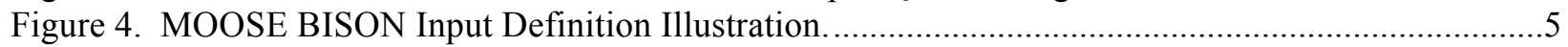

Figure 5. Workbench Validation Panel Illustration Depicting Missing Required Input. ..........................6

Figure 6. Workbench Valid Input Illustration with Satisfied Input Definition..........................................6

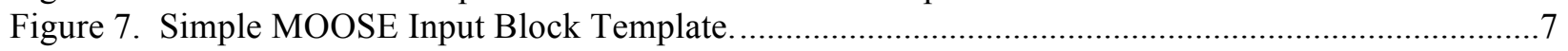

Figure 8. Simple MOOSE Input Sub Block Template.........................................................................

Figure 9. Simple MOOSE Input Named Parameter Template ............................................................

Figure 10. Parameterized MOOSE Input Block Template. ..................................................................

Figure 11. Parameterized MOOSE Input Sub Block Template. .........................................................

Figure 12. Parameterized MOOSE Input Named Parameter Template. ..................................................

Figure 13. Workbench BISON Input Auto-Completion Illustration. ….................................................

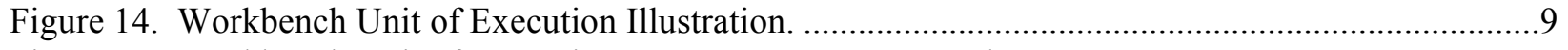

Figure 15. Workbench Unit of Execution MOOSE BISON Example ...................................................9

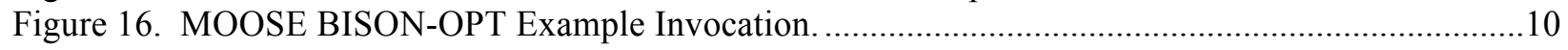

Figure 17. MOOSE BISON Runtime Environment Example Invocation. .............................................11

Figure 18. Workbench MOOSE BISON Runtime Invocation Example. ................................................11 


\section{INTRODUCTION}

The Nuclear Energy Advanced Modeling and Simulation (NEAMS) Workbench is a new initiative for the 2016 fiscal year. It will facilitate the transition from conventional tools to high-fidelity tools by providing a common user interface for model creation, review, execution, and visualization for integrated codes. The Workbench can use common user input by templating engineering scale specifications to applicationspecific input requirements. This will enable multi-fidelity analysis of a system from a common input. The expansion of codes integrated under the Workbench will broaden the NEAMS tools user community and facilitate more science and engineering. This task was officially started in June of 2016 with a focus to support MOOSE applications, with BISON being the pilot application.

\section{MOOSE FRAMEWORK APPLICATION INPUT}

The MOOSE framework uses the GetPot [http://getpot.sourceforge.net/] input file and command line parser. The GetPot project is an anagram of the Linux 'getopt' utility which is used to process program command line arguments. GetPot extends command line argument processing with a hierarchical input format that describes blocks, sub-blocks, and named parameter values of an input. The MOOSE application instantiates an extension of the GetPot utility included in the LibMesh code repository [https://libmesh.github.io/doxygen/getpot_8h_source.html]. GetPot provides constructs that make it easy for users to enter their input into their MOOSE application. The extraction of this user input by the MOOSE application is concise and easy to use.

The MOOSE framework provides a capable input infrastructure that allows MOOSE applications to communicate an input definition, the available blocks, sub-blocks, and named parameters, to the user via a --dump or --yaml command line option. The --dump option produces the input definition in the GetPot format with certain input information (e.g., named parameter legal value enumerations) in comment fields. The --yaml command line option produces a YAML [YAML Ain't Markup Language: http://yaml.org/] formatted input definition. The YAML-formatted input definition provides a more complete input definition for input validation, but it still lacks some important reference information which will be discussed in a later section.

\subsection{GETPOT INPUT PROCESSOR}

GetPot is licensed under GNU Lesser General Public License 2.1, which allows extensions and redistributions. As such, LibMesh has incorporated the GetPot code and has made minor improvements. The GetPot block can contain comments, sub-blocks, and named parameters. The GetPot sub-block can also contain named parameters, comments, and nested sub blocks.

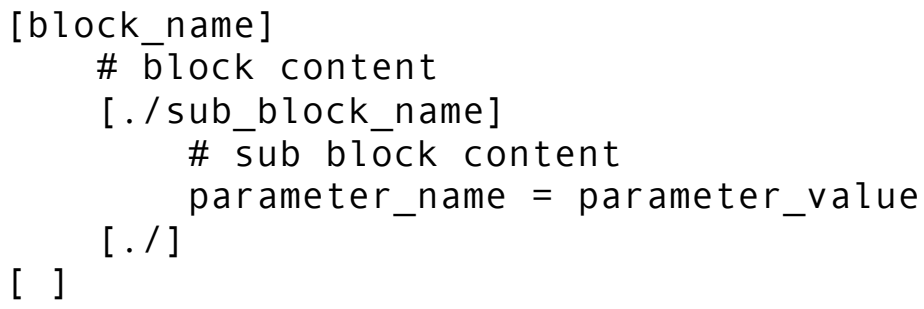

Figure 1. GetPot Block, Sub-Block, and Named Parameter Syntax.

The GetPot API allows for extraction of typed parameter values (integer, real, etc) for easy program integration. The input hierarchy is interpreted as sections, block_name/sub_block_name, which translates reasonably well into familiar Unix file hierarchies. 
The GetPot input format is easy for users to learn, and the GetPot application programming interface (API) is easy to incorporate into $\mathrm{C}++$ programs. However, GetPot input errors are not easily interpreted. Figure 2 depicts a typical syntax error message produced by a MOOSE application using GetPot.

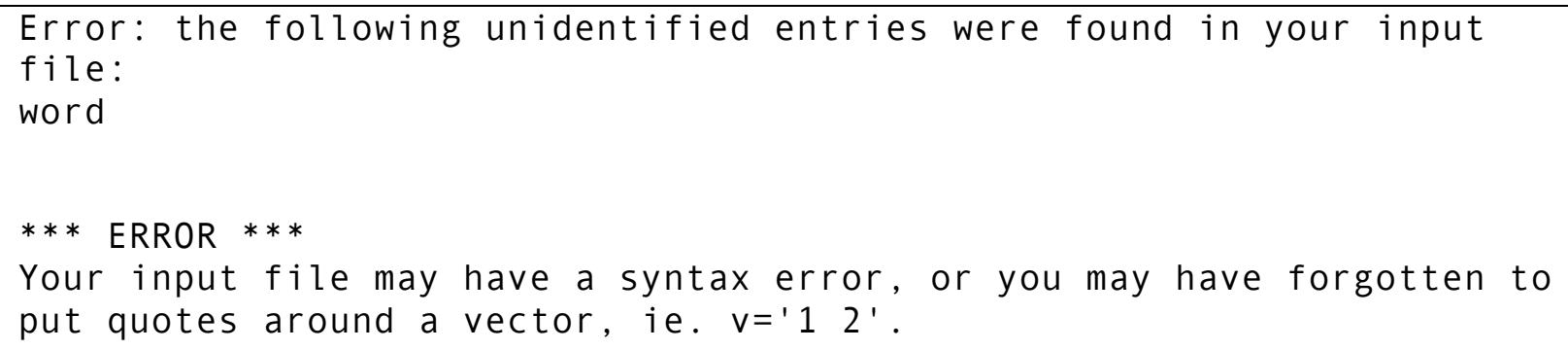

Figure 2. MOOSE application input syntax error message example.

The error message does include the word at fault, but it fails to provide the textual location, line, and column, of the word in the input file. This is not an issue if the fault is unique enough that a simple text search reveals the textual location. However, this does limit programmatic access to the textual location, preventing a user interface from helping facilitate resolution of the issue.

\subsection{NEAMS WORKBENCH INPUT PROCESSOR}

The NEAMS Workbench input processor uses lexicographical analysis - the Lexer-to present words. The Workbench uses the words' textual location to the input parser-the Parser-for semantic analysis. The result is a parse-tree data structure that represents all of the user-specified data, including hierarchy, regardless of correctness. Once the Lexer and Parser have produced a complete understanding of the user input, the validation engine can be invoked for a comprehensive validation check.

It is more complex to implement the Lexer and Parser than the GetPot implementation, but they provide a more robust capability that better facilitates users' input introspection and validation. The increase in program memory as a result of tracking textual location information is deemed worth the computational resources needed to allow effective error communication to the user.

The NEAMS Workbench extracts the textual location of blocks, sub-blocks, and named parameters from the parse-tree, and it presents quick-navigation items which use the textual locations to allow the user to jump to a section of interest. This is made possible by using the section's line and column. This is important regardless of the validity of the user's input, which could be under construction. The input is reprocessed as soon as the user has changed input, allowing quick-navigation items to remain current. Figure 3 depicts the NEAMS Workbench with two BISON input files open. Two input files are shown in the navigation panel, and their sections are listed. Selecting one of these sections will place the Workbench text editor's cursor at the beginning of that section. The section's textual location information is used to highlight where the section begins and ends. 


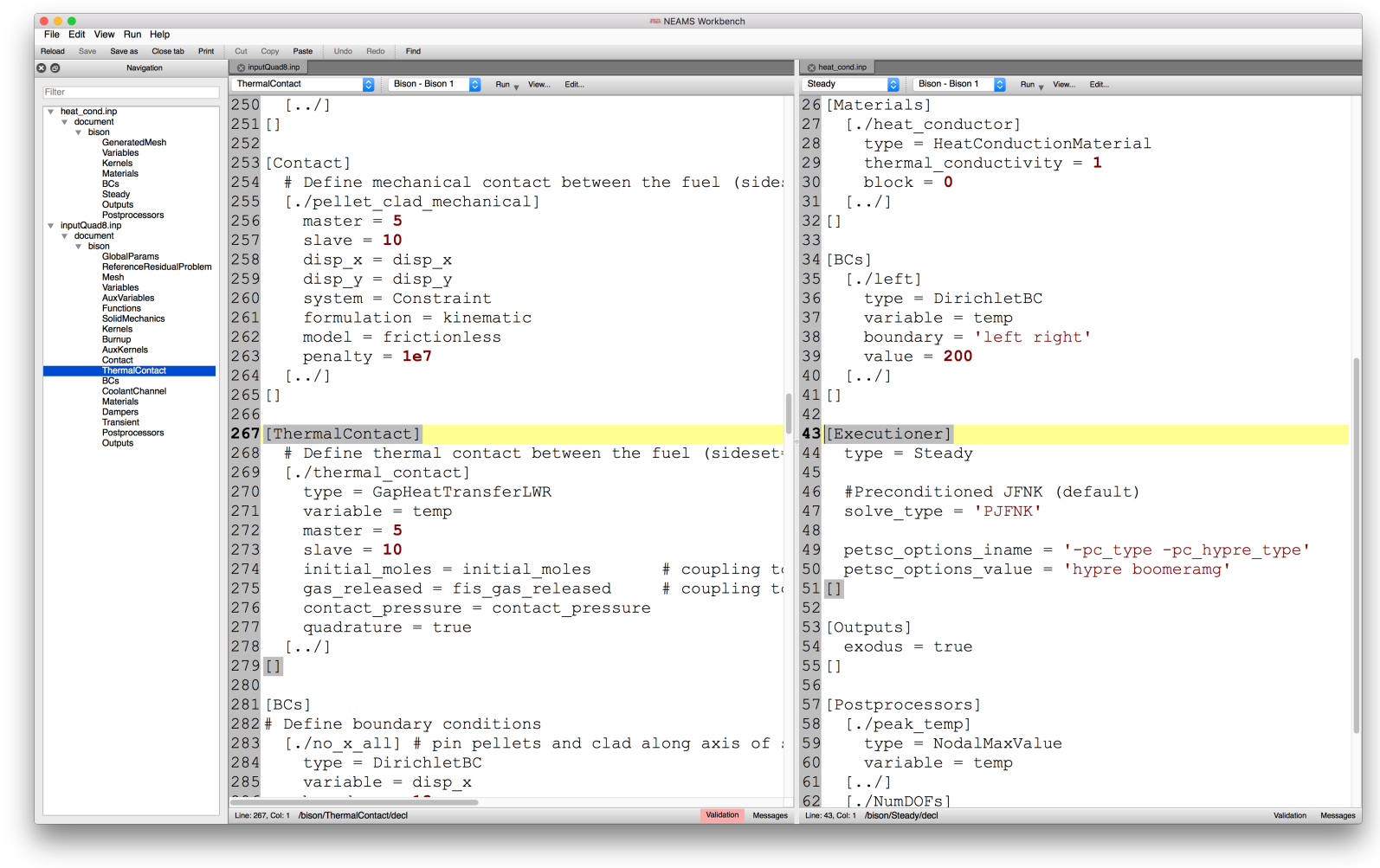

Figure 3. The NEAMS workbench MOOSE BISON input quick navigation.

The section information and textual location information are also used for input validation.

\subsection{NEAMS WORKBENCH INPUT DEFINITION AND VALIDATION}

The NEAMS Workbench Input Validation Engine supports 21 types of input definition rules for validation, but the MOOSE framework input only requires 8 validation rules. These 21 rules have been observed in previously supported input.

1. MinOccurs indicates the minimum occurrence of a component allowed in a context.

2. MaxOccurs indicates the maximum occurrence of a component allowed in a context.

3. ValType indicates the allowed type of a component (e.g., real, integer).

4. ValEnums indicates the allowed enumerated values of a component.

5. MinValInc indicates the minimum inclusive value of a component allowed.

6. MaxValInc indicates the maximum inclusive value of a component allowed.

7. MinValExc indicates the minimum exclusive value of a component allowed.

8. MaxValExc indicates the maximum exclusive value of a component allowed.

9. ExistsIn indicates the context set in which a component must exist.

10. NotExistsIn indicates the context set in which a component must not exist.

11. SumOver indicates the context in which a component must sum to a specified value.

12. SumOverGroup indicates the context and component group in which a component must sum to a specified value.

13. ProdOver indicates the context in which a component must multiply to a specified value.

14. ProdOverGroup indicates the context and component group in which a component must multiply to a specified value. 
15. IncreaseOver indicates the content in which the components values must monotonically be increasing (weakly or strictly).

16. DecreaseOver indicates the content in which the components values must monotonically (weak or strict) be decreasing (weakly or strictly).

17. ChildAtMostOne indicates the set of sub section components from which at most one may exist.

18. ChildExactlyOne indicates the set of sub section components from which exactly one must exist.

19. ChildAtLeastOne indicates the set of sub section components from which at least one must exist.

20. ChildCountEqual indicates the set of sub section components from which the total number of occurrences must be equal to a specified number.

21. ChildUniqueness indicates the set of sub section components from which each component must be unique.

The MOOSE framework input currently only requires MinOccurs, MaxOccurs, ValType, ValEnums, MinValInc, MaxValExc, ExistsIn, ChildAtLeastOne, and ChildUniqueness. The minimum occurrence and maximum occurrence, value type, and value enumeration are rules that deal immediately with the component they are validating and are subsequently easy to understand and implement. The ExistIn, ChildAtLeastOne, and ChildUniqueness rules are more complex, requiring input context facilitated by an XPath [http://www.w3 schools.com/xsl/xpath_syntax.asp]-like query mechanism which heavily relies on paths to components in the input as represented by the parse-tree.

The complete set of rules describing all input is stored in an input definition file commonly referred to as a schema. An ORNL-local MOOSE repository was extended to include a new command line option, --definition, that dynamically produces the schema file containing most information needed for input validation. The schema represents the same hierarchies that are available in the input with the addition of validation rules and metadata such as component description, default, input type and template categorization. The input type and template categorization are used to facilitate input auto-completion of the component from within the Workbench during file content creation. Figure 4 depicts a snippet of MOOSE BISON input definition illustrating the ValType, and ChildAtLeastOne rules and Description metadata field. The type of the variable value within the DirechletBC component must be a String. The ChildAtLeastOne rule indicates that the variable component could be specified in either the GlobalParams, relatively located at ".././GlobalParams/variable/value," or the Di rechletBC component as a subcomponent located at "variable/value. " 


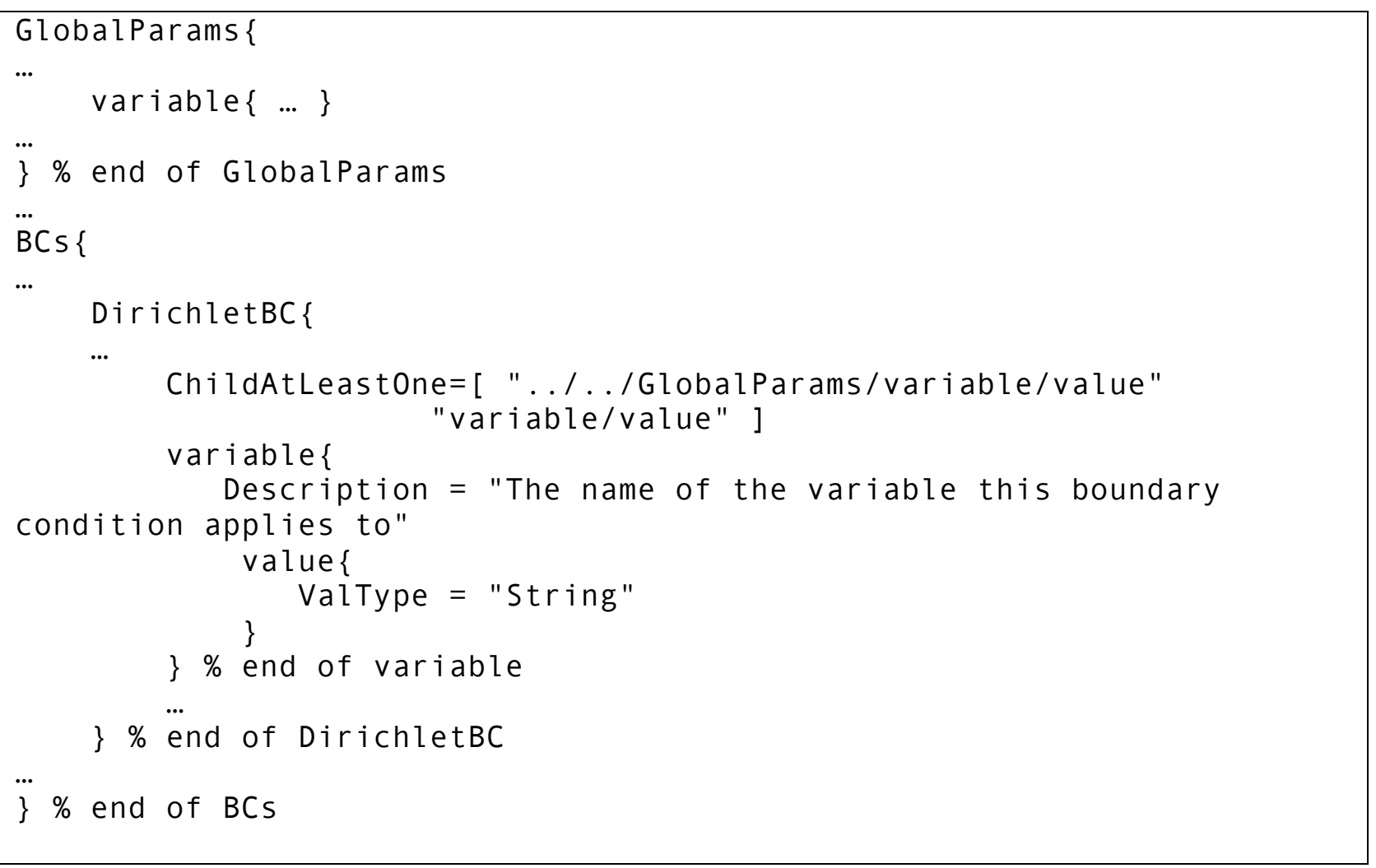

Figure 4. MOOSE BISON input definition illustration.

The input validation engine uses these rules to provide immediate feedback to the user. Figure 5 depicts user feedback driven by the rules illustrated in Figure 4. Because the variable does not exist in the DirichletBC or GlobalParams block, a validation error is presented to the user which reads ' $\mathrm{l}$ ine : 36 column:3 - Validation Error: DirichletBC has zero of:

["../../GlobalParams/variable/value" "variable/value"] - at least one must occur.' Clicking this message in Workbench navigates the user's cursor to the location of the validation error, facilitating quick review and resolution. By defining the var $i$ able named parameter, the input definition is satisfied, and the validation error is no longer presented to the user. The validation error references line 36 , column 3 , which is the textual location of the sub-block containing the validation error. 


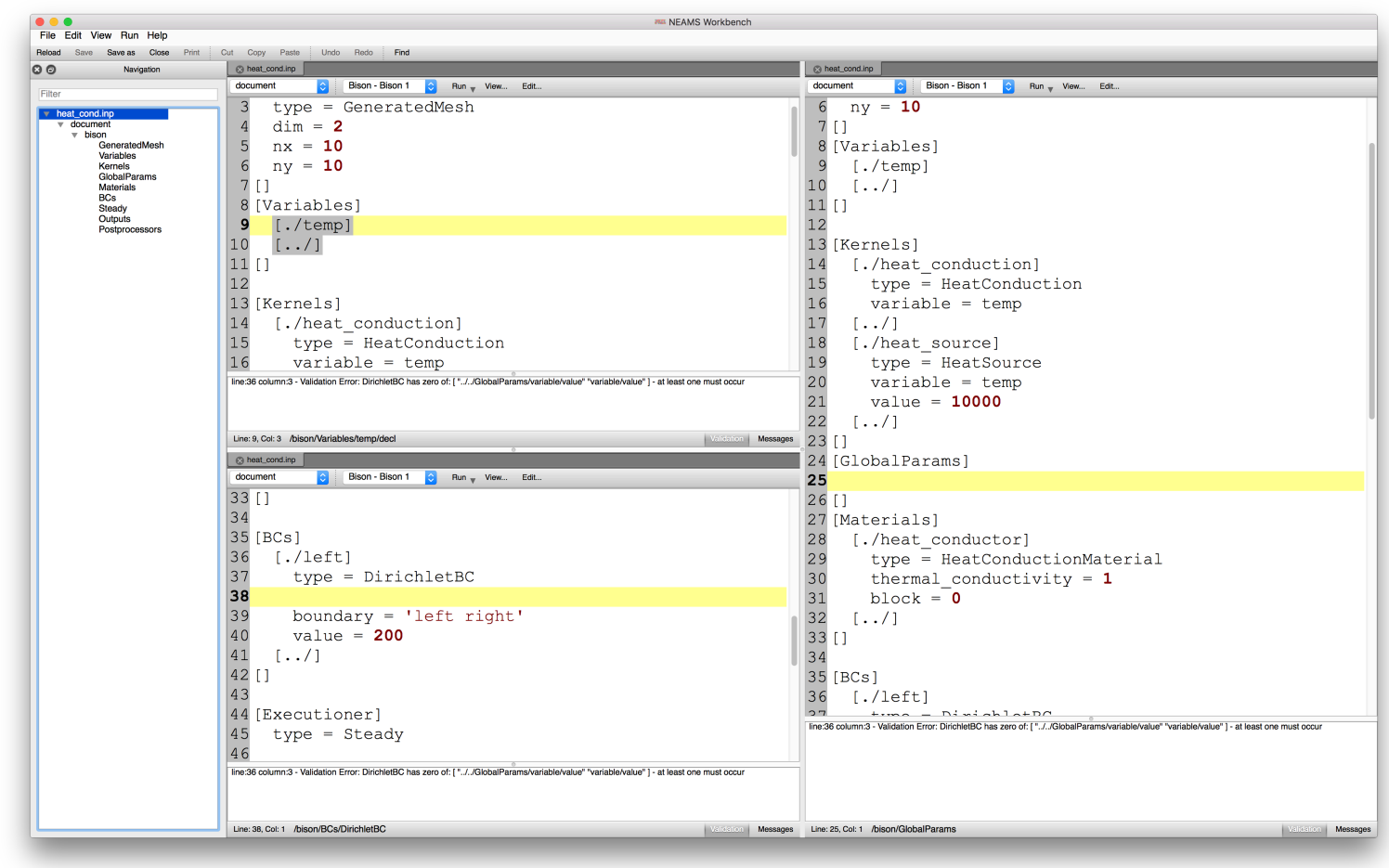

Figure 5. Workbench validation panel showing missing required input.

Figure 6 depicts resolution of the validation error by specifying the variable name parameter in the Global Params block.

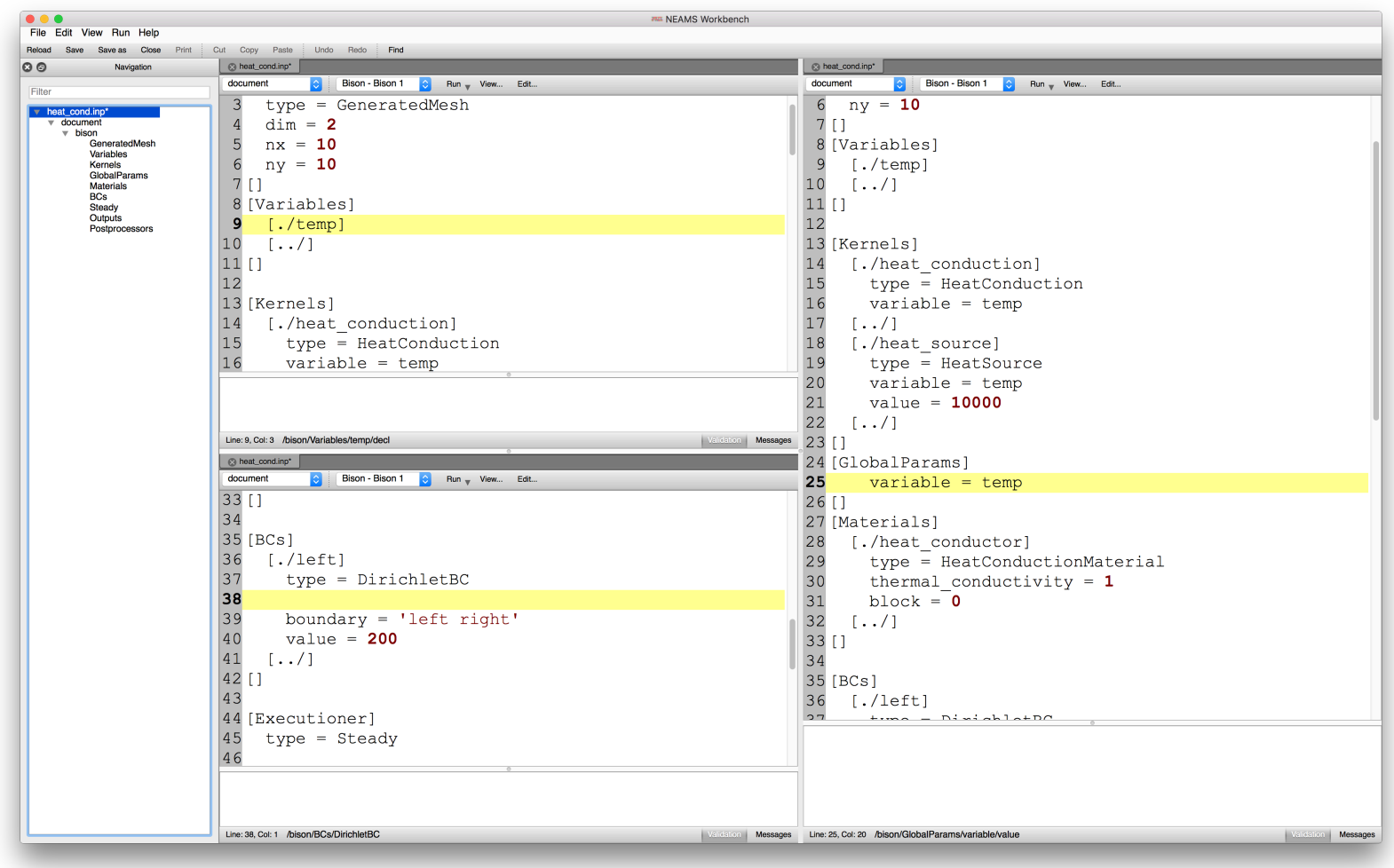

Figure 6. Workbench valid input with satisfied input definition. 


\subsection{NEAMS WORKBENCH MOOSE INPUT CREATION}

At its most basic level, the Workbench is a text editor that makes input creation natural. However, the Workbench can jump start the user with added benefits such as auto-completion of input and input introspection. Input auto-completion uses a template construct in which the template contains placeholders for data of interest. For MOOSE input there is a minimum of three template constructs: the block, the sub-block, and the named parameter. These three templates could be presented as examples with text to be replaced by the user, minimizing the user's required input formatting or required syntax specification. Figure 7 depicts the simplest MOOSE block input template, which requires the user to edit block to be the appropriate block name. Figure 8 depicts the simplest sub-block template, again requiring the user to replace text, sub_block with the appropriate sub-block name. Figure 9 depicts the named parameter MOOSE input template, which requires the user replace both parameter and value with the appropriate name and value of the component.

\section{$[\mathrm{block}]$}

[ ]

Figure 7. Simple MOOSE input block template.

[./sub_block]

$[\ldots /]$

Figure 8. Simple MOOSE input sub-block template.

parameter $=$ value

Figure 9. Simple MOOSE input named parameter template.

The templates shown above aid the uninitiated user in legal syntax of the input, but they do little more. In Figure 9, the effort to replace all but one character slows the user. Preferred templates would contain the respective block, sub-block, and parameter, along with default values listing options available to the user for a given context. To do this, the input definition, in conjunction with the parse-tree and user's input cursor, are required. The user's input cursor provides the textual location, line, and column to Workbench, which can perform an input component lookup into the parse-tree. This lookup provides the input component of focus, subsequently providing the component's path to Workbench. Workbench uses the component's path to conduct the needed lookup into the input definition. The component's input definition communicates all possible input to the Workbench that is available to insert into the file at the user's cursor location. Figure 10, Figure 11, and Figure 12 illustrate the preferred, appropriately parameterized, block, sub-block, and named parameter templates. Workbench provides the $<$ InputName > and < InputValue > attributes to satisfy the template.

\section{$[<$ InputName $>]$}

[]

Figure 10. Parameterized MOOSE input block template.

$[. /<$ InputName $>]$

$[\ldots /]$

Figure 11. Parameterized MOOSE input sub block template.

<InputName $>\quad=\quad\langle$ InputValue $>$

Figure 12. Parameterized MOOSE input named parameter template.

Figure 13 depicts a MOOSE BISON input with an auto-complete list presented to the user illustrating all available components at line 35 . The auto-complete list incorporates the component's name and description, and Workbench uses the input definition for available components in conjunction with the 
parse-tree to filter components as appropriate. For example, components that have already been specified, fulfilling the components maximum occurrence rule, are not available for additional insertion, as it violates the maximum occurrence rule.

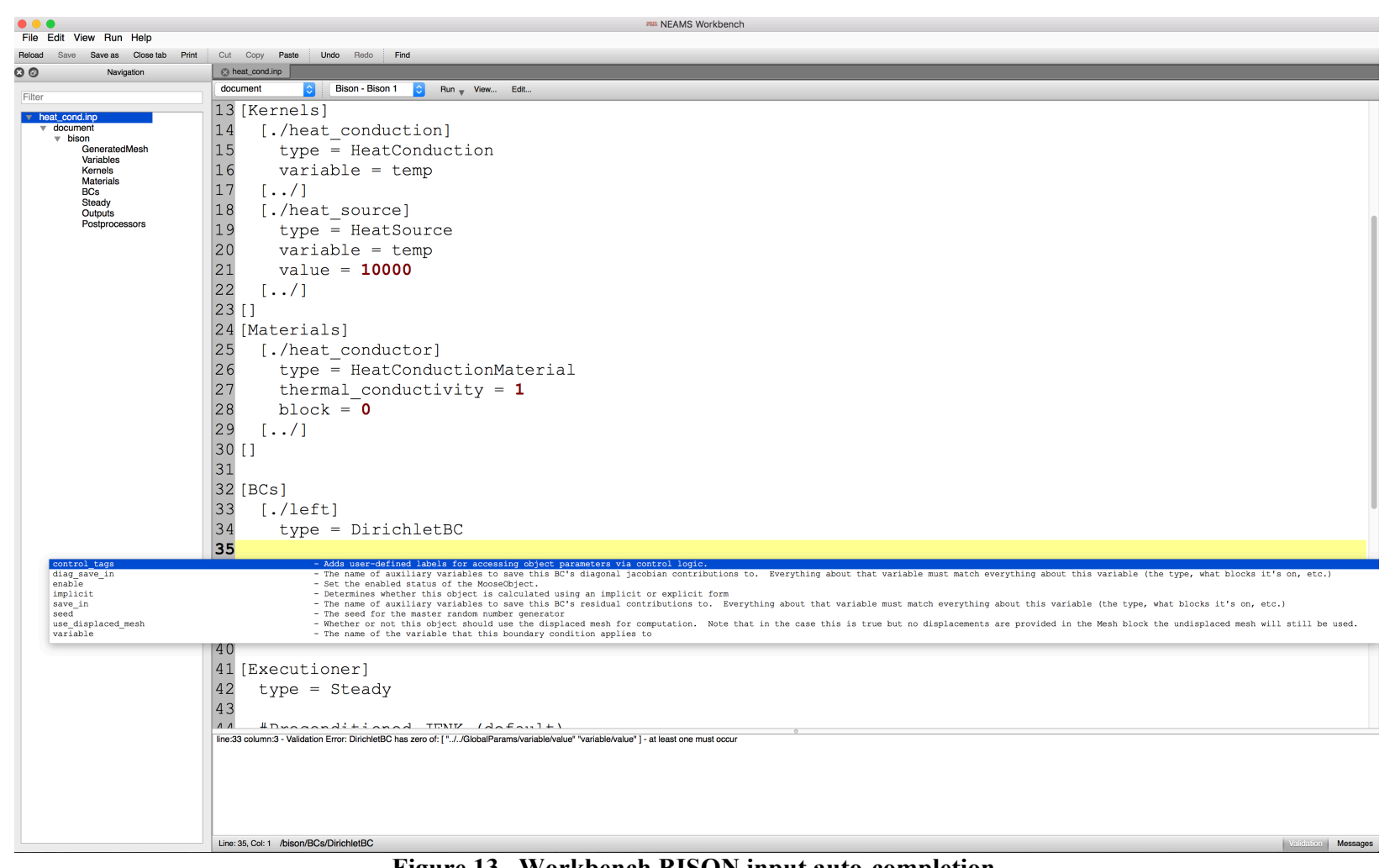

Figure 13. Workbench BISON input auto-completion.

Significant capabilities have been initiated to support MOOSE application input, and there are still areas to be improved, as well as user feedback and significant input features to be captured.

\subsection{NEAMS WORKBENCH MOOSE INPUT CONSIDERATIONS}

MOOSE currently uses the LibMesh extension of GetPot input processor. This input processor lacks the textual location information needed by Workbench to facilitate complex on-demand input validation. The Workbench input processor fulfills these needs, but it duplicates some functional capabilities. In the future, consolidation of these capabilities will facilitate MOOSE and Workbench development teams and will ensure that the user experience remains as consistent as possible.

During an August 2016 collaboration meeting between the MOOSE and Workbench development teams, some deficiencies were recognized in the MOOSE input infrastructure. Subsequently, the appropriate MOOSE development issues were opened and added to a NEAMS Workbench development milestone [https://github.com/idaholab/moose/milestone/4]. Resolution of these issues will facilitate generating a complete schema.

Generation of the input schema from the MOOSE application is an essential capability in facilitating Workbench's understanding of the MOOSE application. The contribution of the ORNL-local MOOSE repository's --definition feature back to the Idaho National Laboratory (INL) master MOOSE repository is needed to facilitate this capability. 
The MOOSE framework greatly facilitates reuse and extension of input components, which allows accelerated application development. However, this has produced a focus on single MOOSE applications. This single application focus causes ambiguity as to which application can run the input. To facilitate the Workbench input generation, validation, and execution, a Unit of Execution construct was added, as depicted in Figure 14.

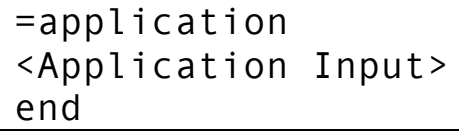

Figure 14. Workbench unit of execution.

Figure 15 illustrates the of the MOOSE BISON application's unit of execution within the Workbench.

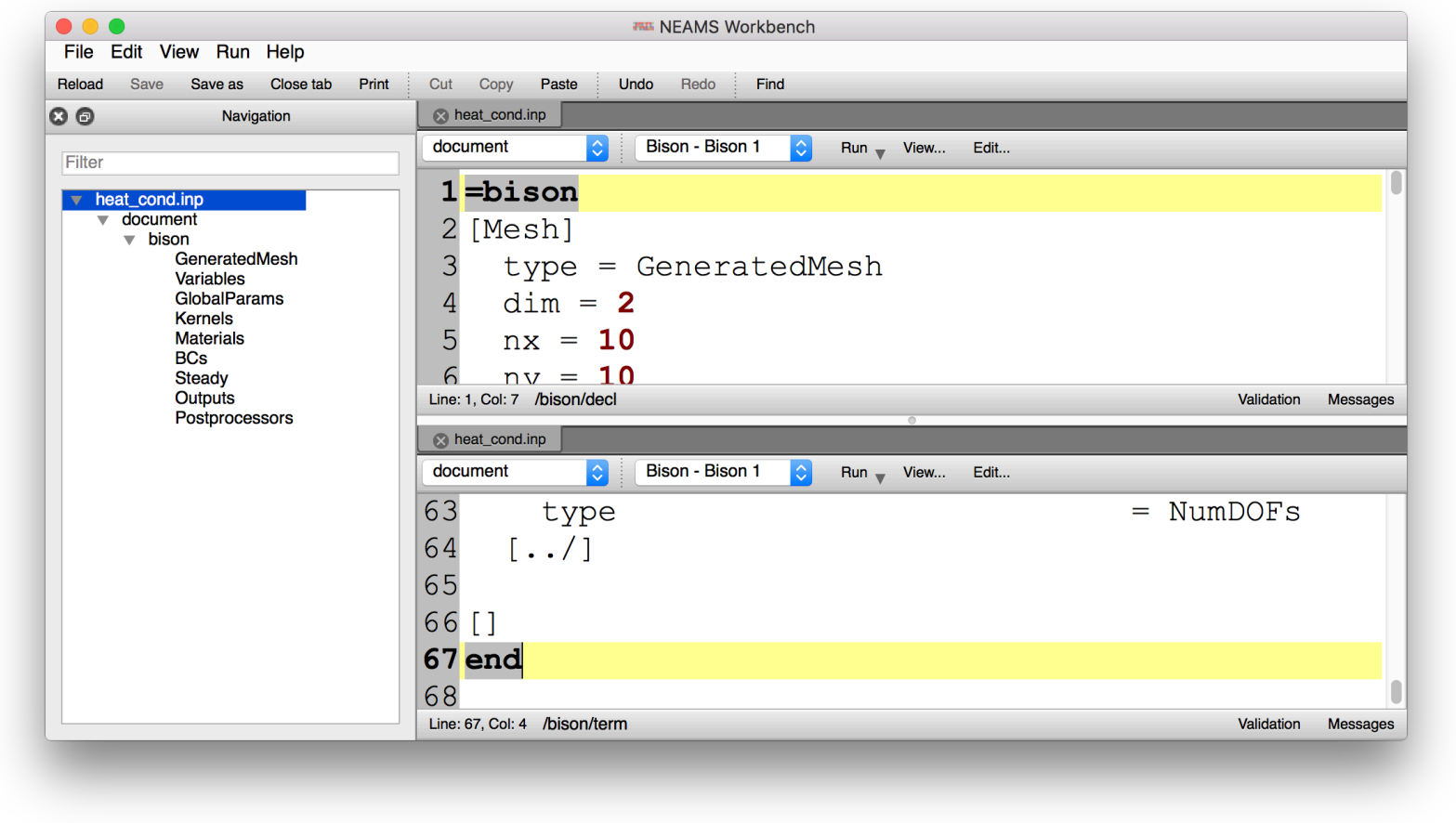

Figure 15. Example Workbench unit of execution for MOOSE BISON.

The unit of execution is accounted for by Workbench at the time of execution by the Workbench runtime environment.

\section{WORKBENCH APPLICATION EXECUTION}

A mission of the Workbench is to facilitate transition from conventional tools to high-fidelity tools. Many of these codes involve different means of invocation, and some have multiple means of invocation. To facilitate execution of these applications, a generic runtime environment interface was created. The intent of the runtime is to provide a consistent interface by which the Workbench can interact with each application in all necessary modes of operation (e.g., serial, parallel, and scheduled execution). The runtime is designed to provide a clean, convenient, consistent means for users to invoke NEAMS toolkit applications via the command line, separate from graphical user interface that is Workbench. 


\subsection{RUNTIME ENVIRONMENT}

After careful consideration, the Python [www.python.org] scripting language was chosen for its combination of cross-platform support, object-oriented design, code readability, and power. The runtime is an object-oriented polymorphic design allowing reuse and extension. A generic base class provides the complete interface to communicate command line options and usage and to facilitate job setup, execution, and finalization of an application's input execution.

Some applications have no runtime environment, requiring the user to manually conduct all steps associated with running the applications. For example, the user might be required to copy the problem input file into a specific location with a specific file name, input,, and then invoke the application, thus producing temporary files and output file(s). This is error prone and should not be important to the user or to Workbench as this logic is application specific. The solution is to contribute a new runtime scriptnew_app.py_to the runtime repository, where the new_app.py overrides the setup, execution, or finalization logic as needed to fulfill the runtime interface. The setup logic might create a working directory, TMPDIR, and then copy the problem. inp into the TMPDIR as TMPDIR/ input. The execution might invoke the application executable, passing application messages back to the calling application (command console, Workbench, etc.). The finalize logic might (1) combine the output files located in TMPDIR into logical order, (2) copy the output back into problem . out, residing next to problem. inp, and (3) delete the TMPDIR to clean up after itself.

Whatever the specific application logic is, there an application.py script will be available to allow consistent invocation and potentially providing great convenience for the application's typical command line user. As the runtime environment matures and additional features are added (queuing system, etc.) for the base class, all incorporated runtimes will benefit.

\subsection{MOOSE BISON RUNTIME ENVIRONMENT}

As of August 2016, the MOOSE BISON application, b i son-opt, was understood to require its invocation to occur in the directory in which the input resides. Figure 16 depicts a typical invocation of the MOOSE BISON application.

/software/neams/moose/bison/bison-opt - i problem. inp

Figure 16. MOOSE BISON-OPT example invocation.

The bi son. py runtime environment encapsulates the bi son-opt command line options, the job setup, execution, and finalize logic with $\sim 200$ lines of Python. Most of these lines are propagating the dozens of command line options through the BISON runtime environment. Figure 17 depicts an example invocation of the BISON runtime environment. Note the complete path to the problem. inp input file. This is not only convenient, but it is also often considered standard. 
/software/neams/rte/bison.py - i /path/to/input/dir/problem.inp

Figure 17. MOOSE BISON runtime environment example invocation.

The Workbench can use this interface to allow users' invocation of multiple MOOSE application inputs in the same interface, as depicted in Figure 18.

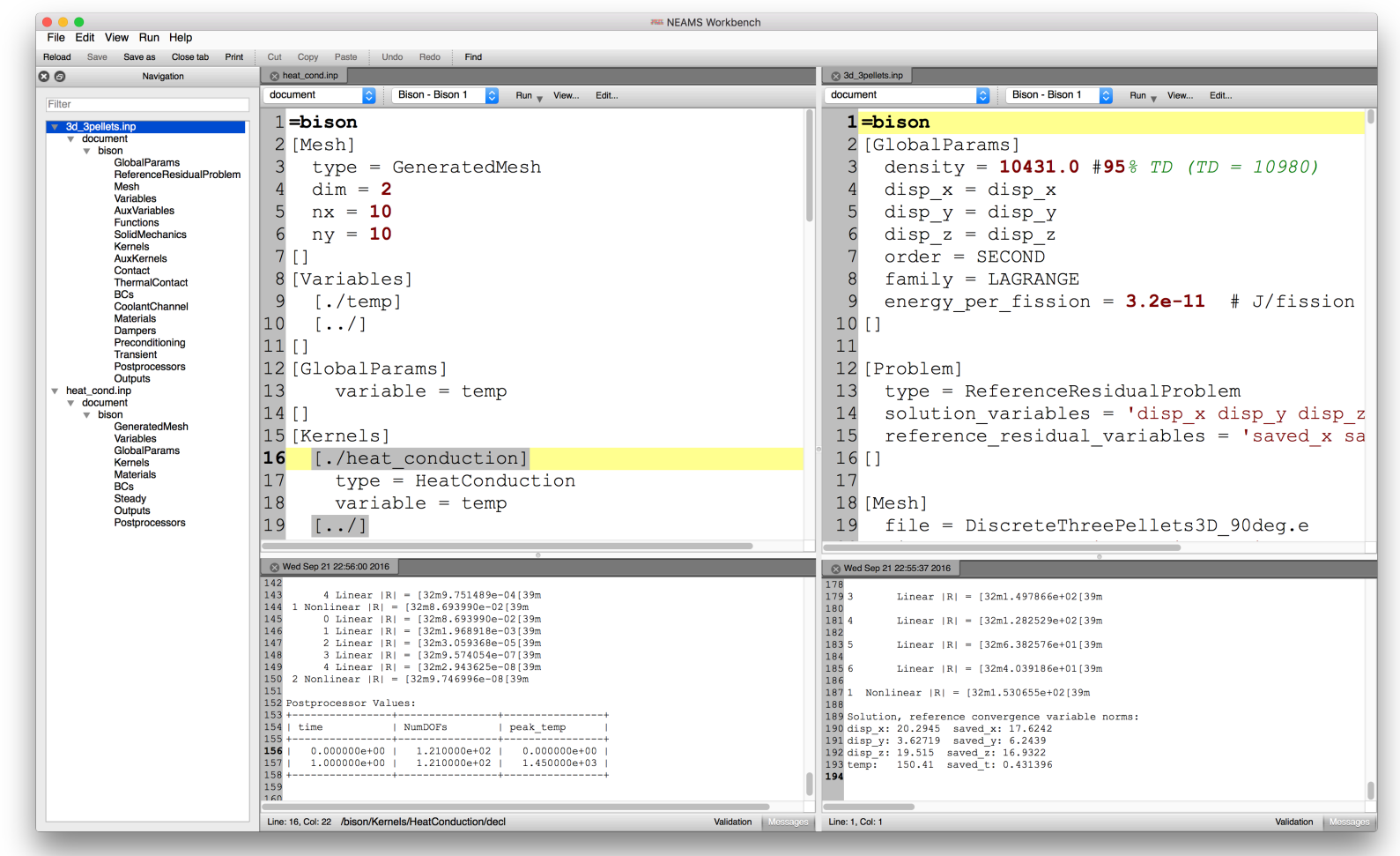

Figure 18. Workbench MOOSE BISON runtime invocation example.

\subsection{FUTURE RUNTIME ENVIRONMENT CONSIDERATIONS}

The runtime environment greatly facilitates the Workbench in its interactions with applications. Current interface has been shown to facilitate local execution of MOOSE BISON applications. With little additional work, more MOOSE applications could easily be added. More work is expected to integrate applications with little or no existing runtime environment. Finally, remote high performance clusters with potential scheduler/queuing interfaces must be designed and integrated to best facilitate large or long-running jobs.

\section{VISUALIZATION INTEGRATION STATUS}

The Workbench development team conducted a review of the LLNL VisIt visualization tool and Kitware Inc., ParaView visualization application APIs. Integration of both into the Workbench is desired. A complicating factor is the tools' common dependency on different versions of Kitware Inc.'s Visualization Tool Kit (VTK). A Workbench and VisIt collaboration meeting was held in August 2016 with Dr. Harinarayan Krishnan of Lawrence Berkeley National Laboratory (LBNL), and it was concluded with a prototype integration of the VisIt Viewer component in Workbench. A collaboration meeting with Bob O'Bara from Kitware, Inc., was held in late September 2016 to discuss the Kitware software solutions, including the ParaView visualization application. 
In addition to the review of the VisIt and ParaView APIs, the MOOSE BISON data and visualization methods were reviewed with staff in CASL and the MOOSE development team. Integration of VisIt and ParaView and streamlining results acquisition and visualization are areas for continued work in FY17.

\section{SUMMARY}

The NEAMS Workbench is a new initiative for the 2016 fiscal year. It is intended to facilitate the transition from conventional tools to high-fidelity tools by providing a common user interface for model creation, review, execution, and visualization for integrated codes. In FY16, the Workbench development team collaborated with Cody Permann and the MOOSE development team at INL to integrate the initial pilot application, MOOSE BISON, into the Workbench. MOOSE BISON input can be created, reviewed, and executed on a local machine. Opportunities for input infrastructure collaboration, improvement in input validation, and results visualization of MOOSE applications still remain and will be addressed in FY17. Due to the design of the MOOSE framework, with little additional effort, additional MOOSE applications can easily be integrated into the Workbench, leveraging the features accomplished in FY16.

To facilitate the broad set of applications to be integrated into the Workbench, an object-oriented, polymorphic Python runtime environment was initiated. This runtime environment provides a consistent interface by which the Workbench or command line user can invoke integrated applications. Additionally, the runtime environment provides a central location by which application invocation conveniences (queuing support) will be centralized.

Visualization integration is still being investigated. In FY16, collaboration meetings were held involving the VisIt visualization tool attended by Dr. Harinarayan Krishnan of LBNL, and ParaView attended by Bob O'Bara of Kitware, Inc. The MOOSE BISON data and visualization methods were reviewed with staff members from CASL and the MOOSE development team. There are opportunities to incorporate existing VisIt and ParaView visualization capabilities into Workbench. Custom VTK capabilities can also be implemented to streamline data analysis workflows that are frequently observed in the application space. 